\title{
Copper(I) iodide supported on modified cellulose-based nano-magnetite composite as a biodegradable catalyst for the synthesis of 1,2,3-triazoles
}

$\underline{\text { Majid M. Heravi }}{ }^{1}$, Samaneh Sabaqian, Firouzeh Nemati, Hossein Taherpour Nahzomi

${ }^{1}$ Department of Chemistry, Faculty of Physics \&Chemistry Alzahra University, Vanak, Tehran, Iran.

\begin{abstract}
Nano-Fe3O4@Cellulose-NH2-CuI as a novel magnetically separable composite was prepared and fully characterized using various techniques including Fourier transform infrared, X-ray photoelectron and energy-dispersive X-ray spectroscopies, X-ray diffraction, field-emission scanning and transmission electron microscopies, thermogravimetric analysis and vibrating sample magnetometry. To obtain an appropriate structure and also to describe to some extent the different kinds of metal-ligand interactions present in the nanoFe3O4@Cellulose-NH2-CuI composite, covalent and electrostatic interactions, density functional theory model chemistry and quantum theory of atoms in molecules method were employed, respectively. This cellulose-based heterogeneous catalyst can effectively promote the one-pot three-component reaction of a variety of terminal alkynes bearing substituted phenyls or propargylic alcohol together with substituted benzyl halides and sodium azide, so-called click reaction, in water to afford the corresponding 1,4-disubstituted 1,2,3-triazoles with improved yields and regioselectivity. The magnetic catalyst was conventionally recovered using an external magnet and reused in at least four successive runs under the optimal reaction conditions, without appreciable loss of its activity.
\end{abstract}

\section{Keyword}

biodegradable support, magnetic composite, metal-ligand interactions, quantum chemistry, Alcohols, Catalysts, Cellulose, Energy dispersive spectroscopy, Ligands, Quantum theory, Thermogravimetric analysis, Transmission electron microscopy, Fourier transform infra reds, Magnetic composites. 